\title{
Structural insight into the Ragulator complex which anchors mTORC1 to the lysosomal membrane
}

\author{
Zongkai Mu${ }^{1}$, Lei Wang ${ }^{1}$, Wei Deng ${ }^{2}$, Jiawei Wang ${ }^{3}$, Geng Wu,* \\ ${ }^{I}$ State Key Laboratory of Microbial Metabolism, School of Life Sciences and Biotechnology, Shanghai Jiao Tong University, \\ Shanghai, China; ${ }^{2}$ National Center for Protein Science Shanghai, Shanghai, China; ${ }^{3}$ State Key Laboratory of Membrane \\ Biology, School of Life Sciences, Tsinghua University, Beijing, China
}

The mechanistic target of rapamycin (mTOR) signal-transduction pathway plays a key role in regulating many aspects of metabolic processes. The central player of the mTOR signaling pathway, mTOR complex 1 (mTORC1), is recruited by the pentameric Ragulator complex and the heterodimeric Rag GTPase complex to the lysosomal membrane and thereafter activated. Here, we determined the crystal structure of the human Ragulator complex, which shows that Lamtor 1 possesses a belt-like shape and wraps the other four subunits around. Extensive hydrophobic interactions occur between Lamtor 1 and the Lamtor2-Lamtor3, Lamtor4-Lamtor5 roadblock domain protein pairs, while there is no substantial contact between Lamtor2-Lamtor3 and Lamtor4-Lamtor5 subcomplexes. Interestingly, an $\alpha$-helix from Lamtor1 occupies each of the positions on Lamtor4 and Lamtor5 equivalent to the $\alpha 3$-helices of Lamtor2 and Lamtor3, thus stabilizing Lamtor4 and Lamtor5. Structural comparison between Ragulator and the yeast Ego1-Ego2-Ego3 ternary complex (Ego-TC) reveals that Ego-TC only corresponds to half of the Ragulator complex. Coupling with the fact that in the Ego-TC structure, Ego2 and Ego3 are lone roadblock domain proteins without another roadblock domain protein pairing with them, we suggest that additional components of the yeast Ego complex might exist.

Keywords: mTORC1 signaling pathway; Ragulator; Rag GTPase complex; Crystal structure; EGO-TC; Lamtor Cell Discovery (2017) 3, 17049; doi:10.1038/celldisc.2017.49; published online 26 December 2017

\section{Introduction}

The mechanistic target of rapamycin (mTOR) signal-transduction pathway regulates many aspects of metabolic processes such as protein translation, lipid synthesis and autophagy in a diverse variety of organisms from yeast to human. Deregulation of the mTOR signaling pathway underlies many human diseases including cancer and diabetes [1-4]. The activation of mTORC1, the central player of the mTOR signaling pathway, which consists of mTOR, Raptor and mLST8, is subject to stringent regulation by the availability of nutrients such as amino acids [5-7]. When amino acids are abundant, mTORC1 is translocated to the lysosomal membrane, through the mediation of the heterodimeric Rag GTPases, which

*Correspondence: Geng $\mathrm{Wu}$

Tel: +86 021 34205914; Fax: +86 021 34207025;

E-mail: geng.wu@situ.edu.cn

Received 10 October 2017; accepted 15 November 2017 consists of RagA or RagB in complex with RagC or RagD [8,9] and the pentameric Ragulator complex $[10,11]$. At the lysosome, the kinase activity of mTORC1 is activated by the small GTPase Rheb, which is also located in lysosomes [12, 13], and then phosphorylates a variety of its target substrates such as eIF4E-binding protein 1 and S6 kinase 1.

The lysosomal membrane-attached Ragulator complex consists of five subunits: Lamtor1, Lamtor2, Lamtor3, Lamtor4 and Lamtor5 (which are also known as p18, p14, MP1, C7orf59 and HBXIP, respectively). The N-terminal region of Lamtor1 contains myristoylation and palmitoylation sites, which anchors the Ragulator complex to the lysosomal membrane [14]. Lamtor2, Lamtor3, Lamtor4 and Lamtor5 all contain roadblock domains [11]. The Rag GTPase complex may interact with the Ragulator complex through the C-terminal domains of RagA/B and $\mathrm{RagC} / \mathrm{D}$, which are also roadblock domains [15]. In addition, it was reported that the Ragulator complex serves as a guanine nucleotide exchange factor for 


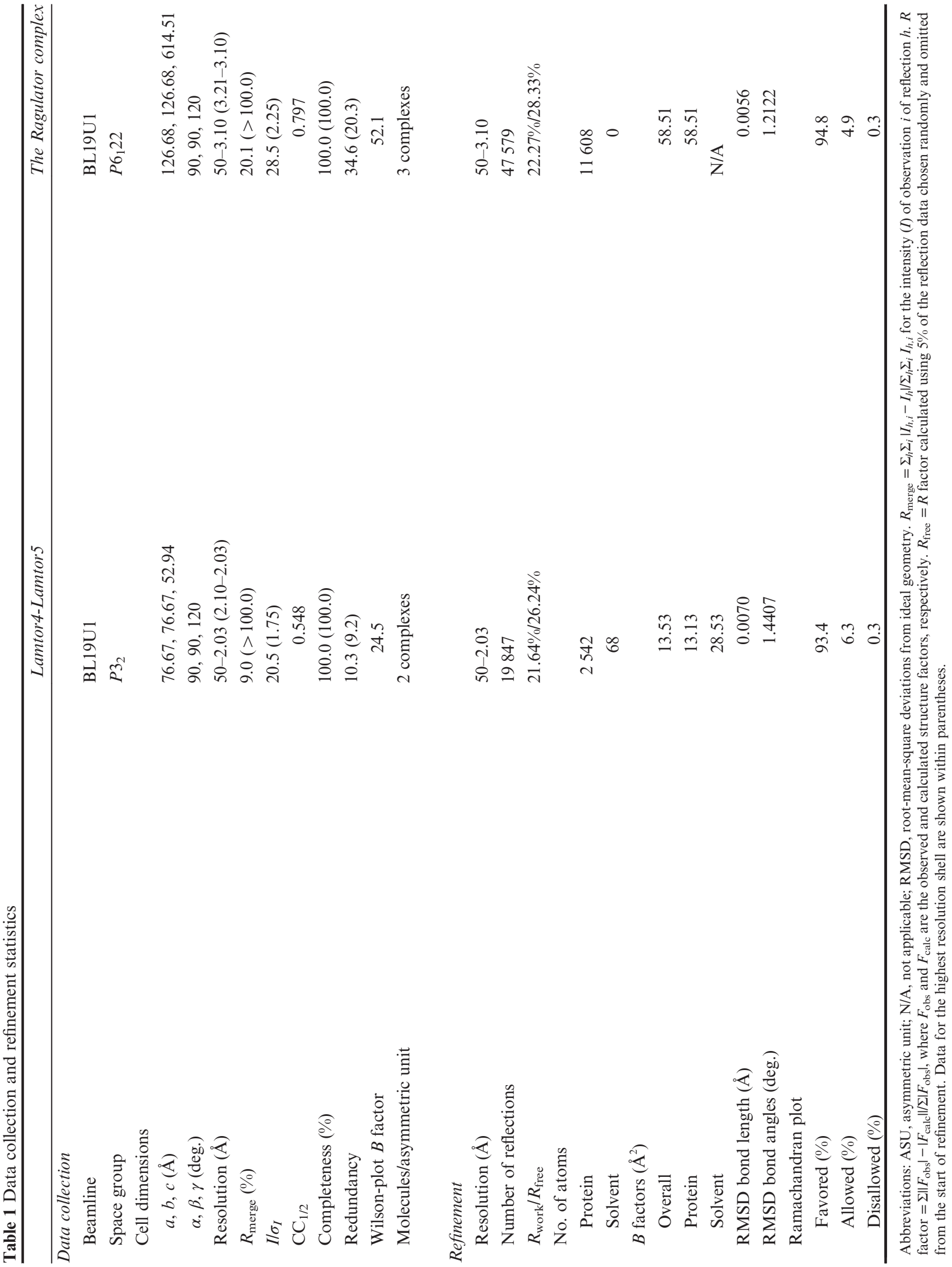


RagA and RagB, promoting their GTP binding in exchange of GDP.

Most of the key players of human mTOR signaling pathway are conserved in yeasts. For example, there are two yeast mTOR homologs, TOR 1 and TOR2 [16]. The yeast counterparts for human $\mathrm{RagA} / \mathrm{RagB}$ and RagC/RagD have been identified to be Gtr1 and Gtr2, respectively[17]. In addition, three yeast proteins, Ego1, Ego2 and Ego3, have been found to interact with each other and might function as components of a Ragulator-like complex (known as Ego-TC presently) in yeasts [18-20]. However, given the fact that human Ragulator is a pentameric complex, whereas yeast EgoTC is only a ternary complex, it is not yet clear all the components of yeast Ragulator-like complex have been identified.

Although the crystal structures of the human Lamtor2-Lamtor3 complex [21, 22] and the yeast Gtr1Gtr2 complex [15, 23] have been reported, the structural assembly of the holo-Ragulator complex still remains elusive. In this work, we determined the crystal structures of both the human Lamtor4-Lamtor5 complex and the human pentameric Ragulator complex. Our structure shows that Lamtor1 exhibits a belt-like structure and wraps around Lamtor2, Lamtor3, Lamtor4 and Lamtor5. There exist extensive interactions between Lamtor1 and all the other four subunits. Lamtor4 associates with Lamtor5 through $\beta$-sheet formation and hydrophobic interactions, similar to the association between Lamtor2 and Lamtor3. Interestingly, both Lamtor 4 and Lamtor5 lack the $\alpha 3$-helix feature which are present in Lamtor2 and Lamtor3. Assembly of the Ragulator complex brings an $\alpha$-helix of Lamtor1 to each of the positions on Lamtor4 and Lamtor 5 equivalent to the $\alpha 3$-helices of Lamtor 2 and Lamtor3, and thus stabilizing Lamtor4 and Lamtor5. In addition, comparison of the structure of the Ragulator complex and that of the previously reported EgoTC indicates that the three components of Ego-TC, Ego1, Ego2 and Ego3, are equivalent to Lamtor1- $\alpha 3$, Lamtor5 and Lamtor2, in terms of their spatial positions. This suggests that other components of the yeast Ragulator-like complex might exist.

\section{Results}

\section{Overall structure of the Ragulator complex}

We first determined the crystal structure of the human Lamtor4-Lamtor5 complex to $2.03 \AA$ resolution by the molecular replacement method, using the structure of the previously reported Lamtor2-Lamtor3 complex as a searching model (Table 1). With the

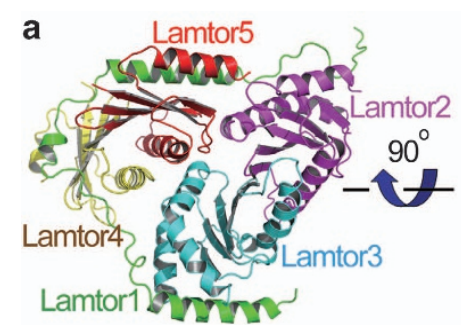

b

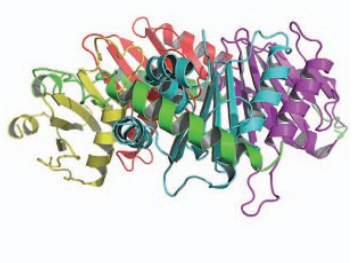

Figure 1 Overall structure of the Ragulator complex. The Lamtor1, Lamtor2, Lamtor3, Lamtor4 and Lamtor5 subunits are colored in green, magenta, cyan, yellow and red, respectively. Each successive panel $(\mathbf{a}, \mathbf{b})$ is rotated as indicated.

structures of the Lamtor2-Lamtor3 and the Lamtor4Lamtor5 complexes as searching models, we then determined the crystal structure of the human pentameric Ragulator complex to $3.01 \AA$ resolution also by the molecular replacement method, with the $R_{\text {work }} /$ $R_{\text {free }}$ factors being $22.27 \% / 28.33 \%$ (Table 1 ).

There are three Ragulator complexes in the crystallographic asymmetric unit (Supplementary Figure S1). However, the Ragulator complex behaves to be monomeric in solution, as assayed by the size exclusion chromatography-multiangle light scattering method (Supplementary Figure S2), suggesting that trimerization of the Ragulator complexes in crystals might result from crystal packing.

In the crystal structure, Lamtorl displays a belt-like shape, and wraps the other four subunits around (Figure 1). Lamtor2 and Lamtor3 form a semiindependent subcomplex, and so are Lamtor4 and Lamtor5. There is little contact between Lamtor2-Lamtor3 and Lamtor4-Lamtor5 subcomplexes, while there are extensive interactions between Lamtorl and both.

\section{Structure of Lamtor 1}

The N-terminal region of Lamtor1 serves as a lipidation site for attaching to the lysosomal membrane and does not participate in the assembly of the Ragulator complex, so we did not include it in our Lamtor1 construct for coexpression with the other four subunits. Although we used Lamtor1 (residues 41-161) for assembling the Ragulator complex and for crystallization, only residues $78-161$ can be seen in the crystal structure. Residues 41-77 of Lamtor1 are possibly disordered in the crystals, and thus do not contribute clearly observable electron density. Lamtor1 contains three $\alpha$-helices, but possesses neither tertiary structure nor hydrophobic core on its own (Figure $2 \mathrm{a}$ and $\mathrm{b}$ ). This provides an explanation for our difficulty of 
a

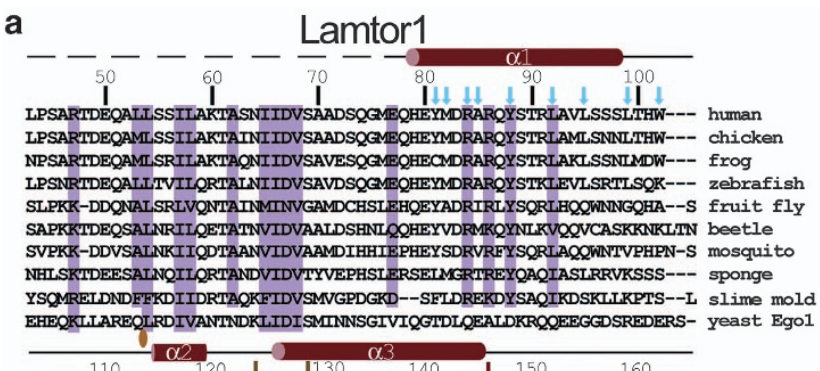

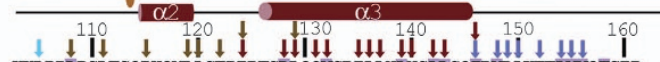
KKLPPIPSLTSQPHQVLASEPIPFSDIQQVSRIAAYAYSALSQIRVDAKRELLVVOFGIP KKLPIIPSLTNQPHQVLASEPVPFTDLQQVSRIAAYAFSALSQIRVDAKKEELVVOFGI KNVPPLPSLTSQPHQILASDPVPFIDIQQVSKIAAYAFSALSQIRVDAKEDLVVOFGIP KPVP-LPSLTSQPHQVLAADLVPHADVQQVSKIAAYAYSAISQIKVDAKBEFLVVOFAIP IAPKGILKDVPSHQFYLS-KPTYYDDTAQMKLFTEKAHISVSHIQIDHKEAVVVPFRIP RLKTCILEDIPWIEITLQSEPISAEDRNIITEALEKAAVAINDIKVEHKEDLVVPERIPC VSHVGIIKDIPNPESVLTSVPISHEDLCAMTRSMVQKANVAVAEIKVKHTTENLVVPFRIP ---A-MPNSDQPPPIALSRELTSSADLKKLLIDRSEATDAAVASMEVKHGEALVVQFAAP

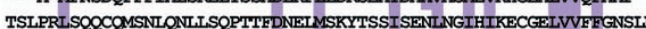
AGDDNLSGHSVPSSGSASPDSAKISKEQLKKLLHSNIINEIFSQSQVNKR PPLTVPF

$\downarrow$ Lamtor2-binding $\downarrow$ Lamtor3-binding $\downarrow$ Lamtor4-binding $\downarrow$ Lamtor5-binding

\section{human} chicken frog zebrafish fruit fly beetle mosquito sponge slime slime mold yeast Egol
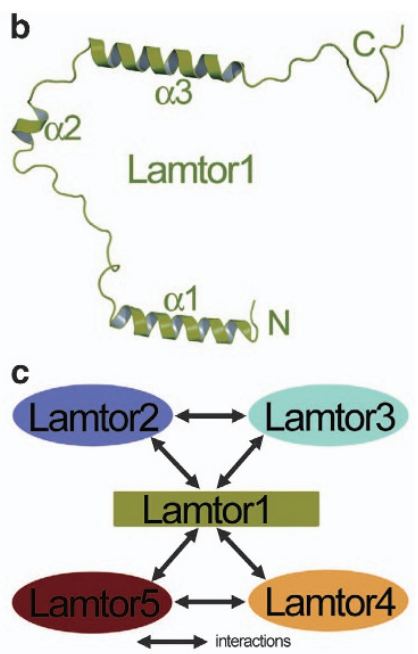

d

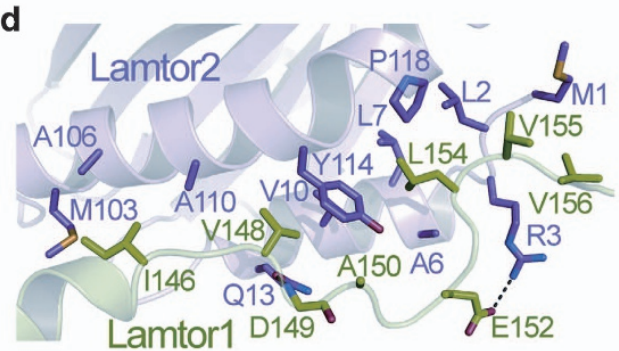

f

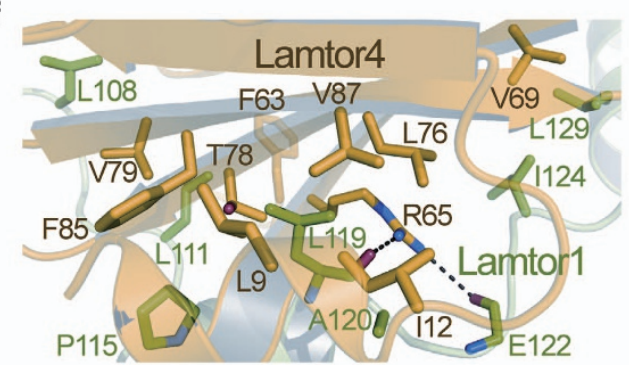

e

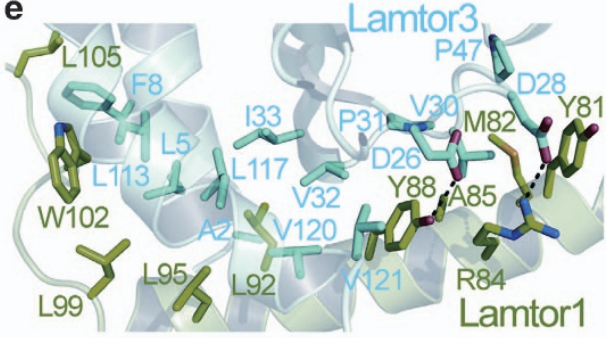

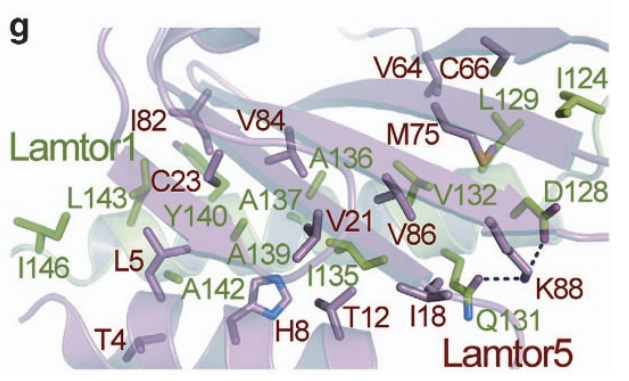

Figure 2 Lamtor 1 does not have a stably folded structure on its own, but adopts an extended conformation and enwraps the other four subunits around. (a) Structure-based sequence alignment of Lamtor1. Secondary structure elements and residue numbers are indicated above the sequence. Conserved residues of Lamtor1 are shaded in pink. Residues of Lamtor1 interacting with Lamtor2, Lamtor3, Lamtor4 and Lamtor5 are indicated by magenta, cyan, brown and red arrows, respectively. The places that contain loop insertions are marked with orange ovals. (b) Lamtor1 adopts an extended conformation and does not have a hydrophobic core on its own. (c) Interactions among the five subunits of the Ragulator complex. Interactions are represented as double-sided arrows. (d) Interaction interface between Lamtor1 (colored in green) and Lamtor2 (colored in magenta). (e) Interaction interface between Lamtor1 (colored in green) and Lamtor3 (colored in cyan). (f) Interaction interface between Lamtor1 (colored in green) and Lamtor4 (colored in yellow). (g) Interaction interface between Lamtor1 (colored in green) and Lamtor5 (colored in salmon). Hydrogen bonds are indicated as black dashed lines.

expressing and purifying good-behaving monomeric Lamtor1 protein by itself (data not shown).

\section{Interactions between Lamtor1 and the other four subunits of Ragulator}

Lamtor1 interacts with all the other four subunits, and is the major contributor of holding up the Ragulator complex together (Figure 2c). It uses its
C-terminal tail to bind to Lamtor2 (Figure 1a and Supplementary Figure S3), uses its $\alpha 1$-helix to associate with Lamtor3 (Figure 1a and Supplementary Figure S4), interacts with Lamtor4 using its $\alpha 2$-helix (Figure 1a and Supplementary Figure S5) and recognizes Lamtor5 through the mediation of its $\alpha 3$-helix (Figure 1a and Supplementary Figure S6). 

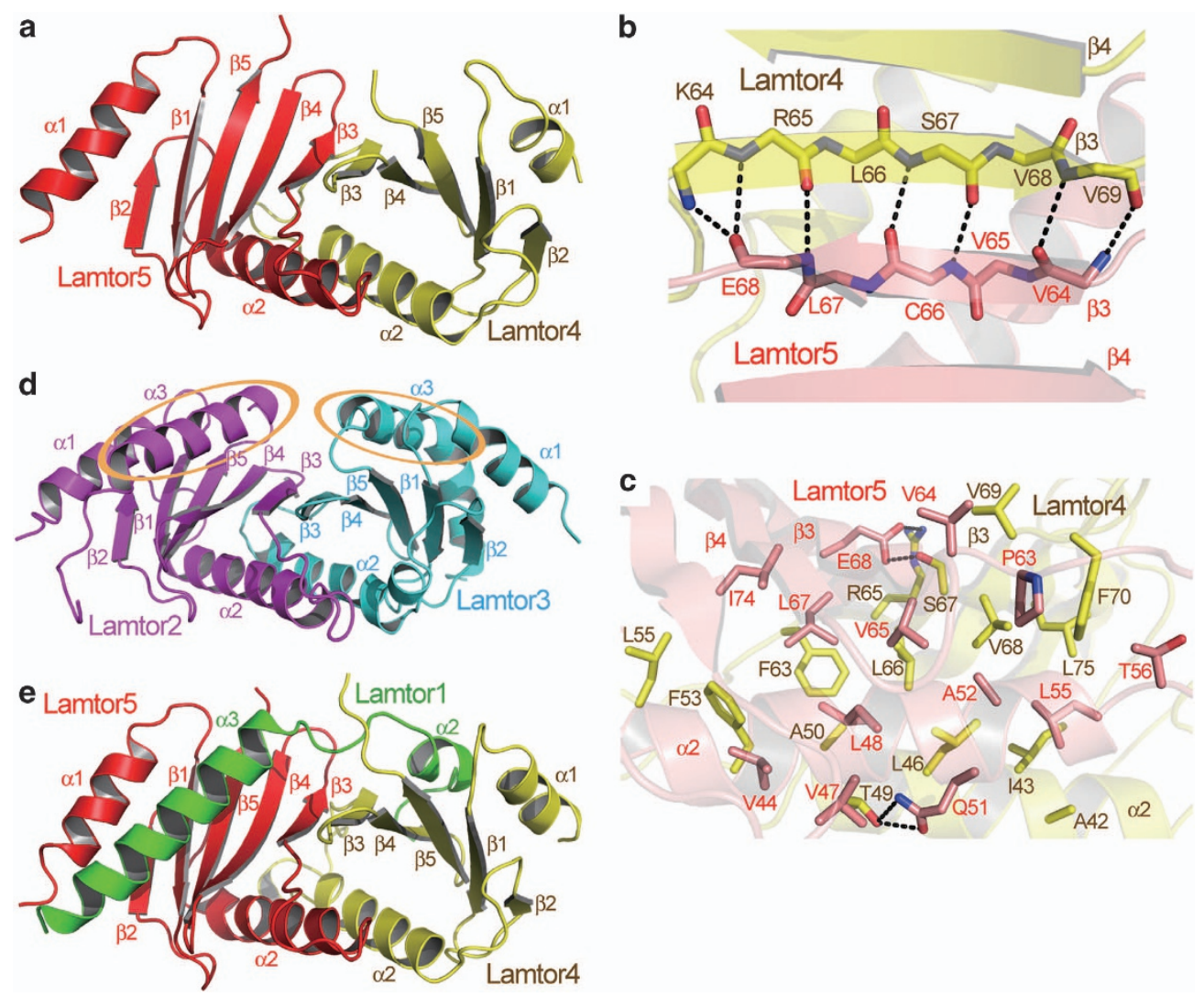

Figure 3 The Lamtor4-Lamtor5 subcomplex is stabilized through association with Lamtor1. (a) Structure of the Lamtor4-Lamtor5 subcomplex. (b, c) Main-chain interactions (b) and side-chain interactions (c) between Lamtor4 and Lamtor5. Carbon atoms of Lamtor4 and Lamtor5 are colored in yellow and salmon, respectively. Hydrogen bonds are indicated as black dashed lines. (d) Structure of the Lamtor2-Lamtor3 subcomplex. Note that both Lamtor4 and Lamtor5 lack the $\alpha 3$-helix features present in Lamtor3 and Lamtor2. (e) The $\alpha 2$ - and $\alpha 3$-helices of Lamtor1 fill in the gaps on Lamtor4 and Lamtor5, respectively, as compared with the Lamtor2-Lamtor3 complex. Therefore, the Lamtor4-Lamtor5 subcomplex is stabilized.

At the Lamtor1-Lamtor2 interface, I146, V148, L154, V155 and V156 of Lamtor1 pack with M1, L2, A6, L7, V10, M103, A106, A110, Y114 and P118 of Lamtor2 through hydrophobic interactions. Besides, the side chain of Lamtor1-E152 forms a salt bridge with that of Lamtor2-R3, and the main chain of Lamtor1-D149 makes hydrogen bond with the side chain of Lamtor2-Q13 (Figure 2a, d and Supplementary Figure S7).

At the interface between Lamtor1 and Lamtor3, Lamtor1 residues Y81, M82, A85, Y88, L92, L95, L99, W102 and L105 at the $\alpha 1$-helix or the loop immediately following $\alpha 1$ make hydrophobic interactions with Lamtor3 residues A2, L5, F8, V30, P31, V32, I33, L113, L117, V120 and V121. In addition, there is electrostatic interaction between Lamtor1-R84 and Lamtor3-D28, and a hydrogen bond between Lamtor1-Y88 and Lamtor3-D26 (Figure 2a, e and Supplementary Figure S8).
For the Lamtor1-Lamtor4 contacting interface, there exist hydrophobic interactions between Lamtor1L108, -L111, -P115, -L119, -A120, -I124, -L129 and Lamtor4-L9, -I12, -F63, -V69, -L76, -T78, -V79, -F85, -V87. Moreover, the main chains of Lamtor1-L119 and -E122 form a couple of hydrogen bonds with the guanidinium group of the side chain of Lamtor4-R65 (Figure 2a, f and Supplementary Figure S9).

At the binding interface between Lamtorl and Lamtor5, extensive hydrophobic interactions occur between residues I124, L129, V132, I135, A136, A137, A139, Y140, A142, L143 and L146 on the $\alpha 3$-helix of Lamtor1 and residues T4, L5, H8, T12, I18, V21, C23, V64, C66, M75, I82, V84 and V86 on the $\alpha 1$-helix and the $\beta 1, \beta 3, \beta 4$ and $\beta 5$ strands of Lamtor5. Meanwhile, the side-chain amide group of Lamtor1-Q131 and the side-chain carboxyl group of Lamtor1-D128 form charge-stabilized hydrogen bonds with the amino group of Lamtor5-K88 side chain (Figure 2a, g and Supplementary Figure S10). 

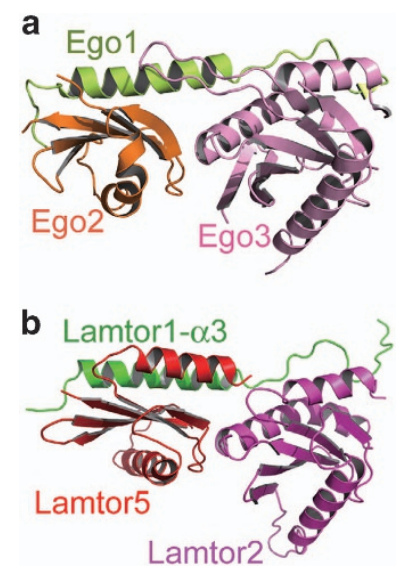

Figure 4 The Ego-TC complex corresponds to half of the Ragulator complex. (a) Structure of the previously reported EgoTC complex. The Ego1, Ego2 and Ego3 subunits are colored in limon, orange and pink, respectively. (b) Structure of the Lamtor1a3-Lamtor5-Lamtor2 subcomplex, which is highly similar in the overall assembly of the Ego-TC complex. Lamtor1- $\alpha 3$, Lamtor5 and Lamtor2 are colored in green, red and magenta, respectively, and their spatial positions correspond to those of Ego1, Ego2 and Ego3, respectively.

Lamtor1 stabilizes Lamtor4 and Lamtor5 by filling up the gaps corresponding to the positions of the $\alpha 3$-helices of Lamtor2 and Lamtor3

Within the Ragulator complex, Lamtor4 and Lamtor5 form a subcomplex (Figure 3a), through $\beta$-sheet formation between their $\beta 3$ strands (Figure 3b), as well as extensive hydrophobic interactions between their $\alpha 2$ helices as well as $\beta 3$ and $\beta 4$ strands (Figure 3c). The mode of their interaction is very similar to that of the interaction between Lamtor2 and Lamtor3 (Supplementary Figure S11).

Interestingly, both Lamtor4 and Lamtor5 only possess two $\alpha$-helices, whereas Lamtor 2 and Lamtor3 contain three. Comparison between the Lamtor4Lamtor5 subcomplex and the Lamtor2-Lamtor3 subcomplex shows that Lamtor4 and Lamtor5 lack the feature of the $\alpha 3$-helix, which is present in both Lamtor2 and Lamtor3 (Figure 1a and d, note the orange ovals in Figure 1d). On the other hand, within the Ragulator complex, an $\alpha$-helix from Lamtor 1 fills in each of the gaps on Lamtor4 and Lamtor5 corresponding to the positions of $\alpha 3$-helices of Lamtor2 and Lamtor3: the $\alpha 2$-helix of Lamtor1 fills in the gap on Lamtor4, whereas the $\alpha 3$-helix of Lamtor 1 fills in the gap on Lamtor5 (Figure 3d). In this way, the Lamtor4-Lamtor5 subcomplex is stabilized by Lamtor1, and by their assembly into the Ragulator complex.
The yeast Ego-TC complex only corresponds to half of the human Ragulator complex

The Ego-TC, comprising of Ego1, Ego2 and Ego3, has been identified to be the yeast counterpart of the Ragulator complex [16-18]. In the structure of the Ego-TC complex, Egol contains a single long $\alpha$-helix and a short $\beta$ strand, and interacts with both Ego2 and Ego3, which are also roadblock domaincontaining proteins. On the other hand, there is no contact between Ego2 and Ego3 (Figure 4a). A comparison between the Ego-TC and Ragulator structures reveals that the overall assembly of EgoTC as well as the spatial position of every one of its subunits is strikingly similar to those of half of the Ragulator complex. Egol corresponds to the C-terminal half of Lamtor1 including its $\alpha 3$-helix and the C-terminal tail, Ego2 is equivalent to Lamtor5 and $\mathrm{Ego} 3$ is the counterpart of Lamtor2 (Figure 4b). The C-terminal half of Lamtor1 also links Lamtor5 and Lamtor2 together, which do not have direct association with each other. Considering the fact that Ragulator possesses five subunits while Ego-TC only has three, as well as the fact that roadblock domain proteins usually exist in pairs such as the Lamtor2Lamtor3 pair and the Lamtor4-Lamtor5 pair, it is very probable that additional components of the Ego complex remain to be discovered.

Mutating or deleting the C-terminal conserved LVVxF motif of Lamtorl does not affect the interaction between Ragulator and the Rag GTPase complex in vitro

One of the major functions of the Ragulator complex is to serve as a lysosome anchor, which recruits the heterodimeric Rag GTPase and its associated mTORC1 complex to the lysosomal surface [10]. The N-terminal region of Lamtor1 contains the lipidation sites [10] and is not supposed to be involved in associating with the Rag GTPase complex. Indeed, purified Ragulator complex with residues (1-40) of Lamtor1 deleted associated with the $\mathrm{RagA} / \mathrm{RagC}$ complex, as demonstrated through the $\mathrm{Ni}^{2+}$-NTA affinity chromatography assay (Figure 5a and Supplementary Figure S13a). The C-terminal domains of both $\mathrm{RagA}$ and $\mathrm{RagC}$ are roadblock domains, and have been previously suggested to mediate the association with the Ragulator complex [15]. In support of this notion, purified RagA-CTD/ RagC-CTD complex interacted with the Ragulator complex (Figure $5 \mathrm{~b}$ and Supplementary Figure S13a).

Examination of the Ragulator structure shows that the C-terminal part of Lamtor1, especially residues 
a

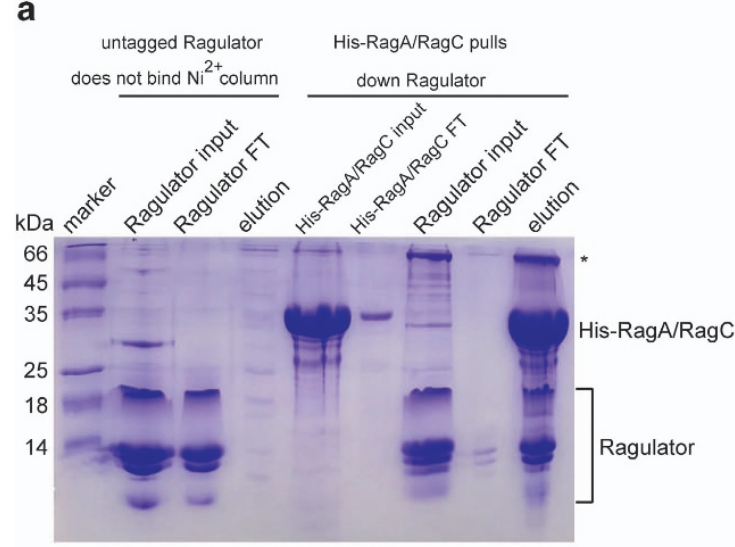

C

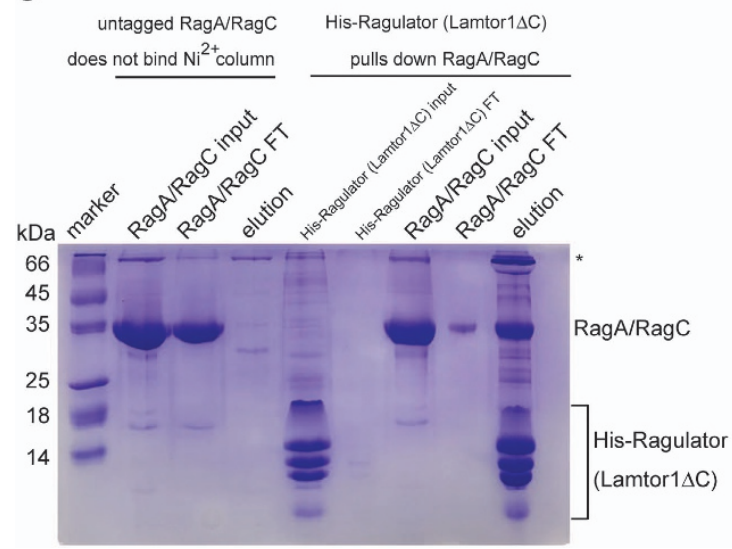

b

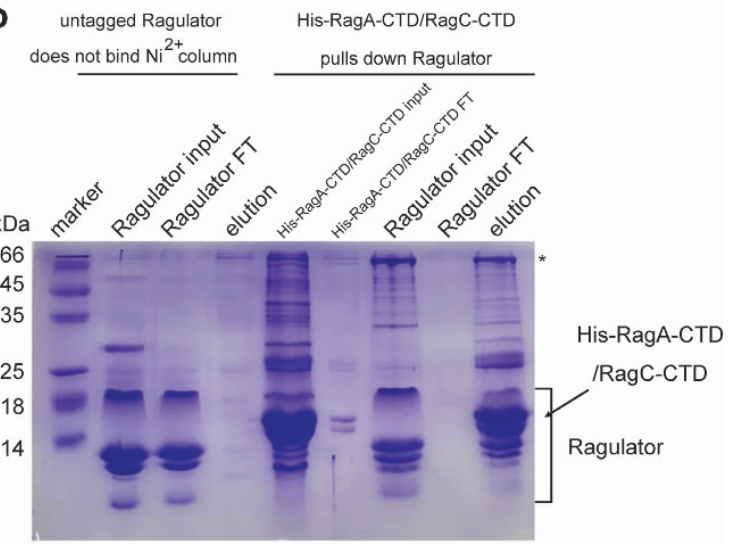

d

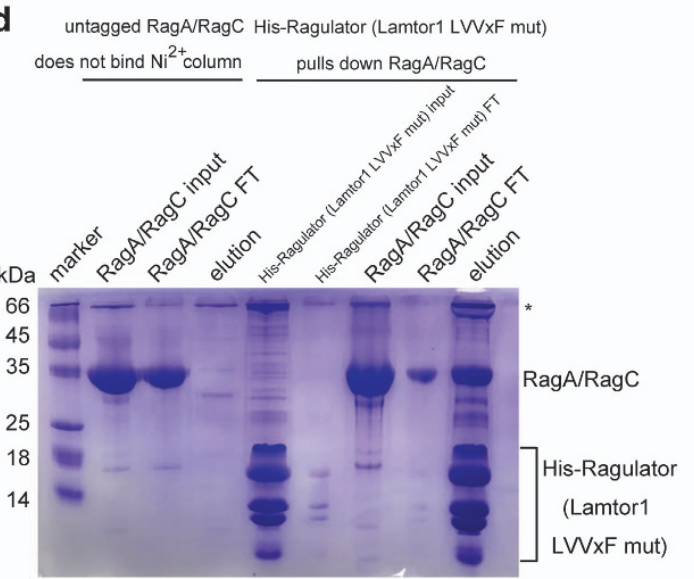

Figure 5 Mutation or deletion of the C-terminal conserved LVVxF motif of Lamtor1 did not affect the association between Ragulator and the RagA-RagC GTPase complex in vitro. (a) Our purified Ragulator complex interacted with the RagA-RagC complex, as examined by the $\mathrm{Ni}^{2+}$-NTA affinity chromatography assay. (b) The Ragulator complex also associated with the RagACTD-RagC-CTD complex. (c) Deletion of residues 151-161 of Lamtor1 did not apparently disrupt the complex formation between Ragulator and the RagA-RagC complex. (d) Point mutation of residues $\mathrm{L}^{154} \mathrm{VVQF}{ }^{158}$ to $\mathrm{D}^{154} \mathrm{DDQD}^{158}$ also did not substantially affect the binding between Ragulator and the RagA-RagC complex. The '*' symbol stands for a nonspecific band copurified with our target proteins. FT, flow-through.

$\mathrm{L}^{154} \mathrm{VVQF}^{158}$, is solvent exposed and is among the most conserved parts of the Ragulator surface (Figure 2a and Supplementary Figure S12). To investigate the functional importance of this motif, we either deleted residues $151-161$ of Lamtor1 or mutated $\mathrm{L}^{154} \mathrm{VVQF}^{158}$ to $\mathrm{D}^{154} \mathrm{DDQD}{ }^{158}$, and investigated whether the resulting Lamtor1 mutants could still support the association between Ragulator and the Rag GTPases. To our surprise, deleting or mutating the highly conserved C-terminal region of Lamtorl did not appreciably affect the Ragulator-Rag complex formation (Figure 5c, d and Supplementary Figure S13b). Presumably, other subunits of the Ragulator complex besides Lamtor1, such as Lamtor2 and Lamtor3, contribute to the binding between Ragulator and the Rag GTPases.

\section{Discussion}

The pentameric Ragulator complex plays a critical role in the mTOR signal-transduction pathway by bringing the mTORC1 complex to the lysosomal surface through the mediation of the Rag GTPases, thus mTORC1 can be activated by the lysosome-located small GTPase Rheb. Our structure of the Ragulator complex reveals that Lamtor1 possesses a belt-like shape and encircles the two subcomplexes, Lamtor2Lamtor3 and Lamtor4-Lamtor5. Extensive associations, predominantly being hydrophobic interactions, occur between Lamtor 1 and every one of the other four subunits. Interestingly, two helices from Lamtorl fill up the gaps on Lamtor4 and Lamtor5, which correspond to the positions of the $\alpha 3$-helices of Lamtor 2 and 
of Lamtor3, thereby stabilizing the Lamtor4-Lamtor5 subcomplex.

A comparison between the Ragulator pentameric complex and the Ego-TC trimeric complex shows that Ego-TC only accounts for half of the Ragulator complex. Ego1, Ego2 and Ego3 are equivalent to the C-terminal half of Lamtor1, Lamtor5 and Lamtor2, respectively. As a rule of thumb, roadblock domain proteins usually exist in pairs, such as the Lamtor2Lamtor3 pair, the Lamtor4-Lamtor5 pair and the RagACTD-RagC-CTD pair. In the roadblock domain protein pairs, their central $\beta$-sheets merge together to form a more extensive $\beta$-sheet, thus stabilizing each other. However, in the Ego-TC complex, the roadblock domain proteins Ego2 and Ego3 have no contact with each other and apparently exist as two lone roadblock domains. These facts prompt us to propose that there might be additional components of the Ego complex. For example, there might be a roadblock domain protein pairing with Ego2, and another roadblock domain protein pairing with Ego3. Interestingly, it has been suggested previously that Ego3 might function as a homodimer to mediate the interaction between the Gtr1Gtr2 complex and Egol so as to activate the TORC1 complex [18]. Considering the similarity between Ego2 and Ego3, it is quite likely that Ego2 also forms a homodimer. Therefore, in addition to the hypothesis of there existing additional components of the Ego complex, there lies another possibility that Ego1, the Ego2 homodimer and the Ego3 homodimer assemble into a pentameric Ego1-(Ego2) $)_{2}$-(Ego3) $)_{2}$ complex. Presumably, the N-terminal region of Egol, which was disordered and could not be observed in the crystal structure of the Ego-TC complex [19], interacts with these additional roadblock domain components of the Ego complex. In this way, Ego1 enwraps Ego2, Ego3 and the additional roadblock domain components together, as Lamtor1 does in the Ragulator complex.

In the mean time when this manuscript was being prepared, a report on the crystal structures of Ragulator and the Ragulator-RagA-CTD-RagC-CTD complex was published by Scheffzek and co-workers [24]. The structural assembly of the Ragulator complex in their report was similar to ours. However, they found that mutation or deletion of the $\mathrm{C}$-terminal $\mathrm{L}^{154} \mathrm{VV}^{156}$ motif of Lamtor1 disrupted the association between Ragulator and the Rag GTPase complex, both by the coimmunoprecipitation and by the immunofluorescence approaches. The discrepancy between our result and theirs might be due to the fact that we used purified recombinant protein to perform the interaction assay in vitro, whereas they used in vivo methods to examine protein interactions in cells. It is likely that there exist post-translational modifications on components of Ragulator or the Rag GTPase complex besides lipidation of the $\mathrm{N}$ terminus of Lamtor1, so that the interaction between Ragulator and the Rag GTPase complex is weakened compared with unmodified proteins as we have used. Certainly, other possibilities might also exist which await further investigations.

\section{Materials and Methods}

\section{Purification and crystallization of the Lamtor4-Lamtor5 complex}

The cDNAs of full-length human Lamtor4 and residues 83-173 of human Lamtor5 were subcloned into a pRSFDuet-1 vector (Novagen, Darmstadt, Germany) with a tobacco etch virus (TEV) protease cleavage site between the $6 \times$ His tag and the $\mathrm{N}$ terminus of Lamtor 5 for recombinant protein expression in the Escherichia coli strain BL21(DE3). The Lamtor4Lamtor5 protein complex was purified using $\mathrm{Ni}^{2+}$-NTA affinity chromatography (GE Healthcare, Little Chalfont, UK), and the eluted protein was incubated with the TEV protease (1:50 ratio, $\left.\mathrm{w} \mathrm{w}^{-1}\right)$ at $4{ }^{\circ} \mathrm{C}$ for $12 \mathrm{~h}$ to remove the $6 \times$ His tag in the presence of $\beta$-mercaptoethanol $\left(1: 1000\right.$ ratio, $\left.\mathrm{Vv}^{-1}\right)$. The protein was further purified by anion exchange chromatography, followed by gel filtration chromatography in $10 \mathrm{~mm}$ Tris- $\mathrm{HCl}, \mathrm{pH}$ 8.0, $100 \mathrm{~mm} \mathrm{NaCl}$ and $2 \mathrm{~mm}$ dithiothreitol. The crystals were obtained using the hanging-drop vapordiffusion method with reservoir solution containing $0.1 \mathrm{M}$ Tris- $\mathrm{HCl}, \mathrm{pH} 8.0,3.5 \mathrm{~m}$ sodium formate at $287 \mathrm{~K}$ for 2 days.

\section{Purification and crystallization of the Ragulator complex}

Three two-promoter coexpression vectors were used to coexpress the five subunits of Ragulator complex. Lamtor1 (residues 41-161) was cloned into the multiple cloning sites 1 of pETDuet-1 (Novagen) with a TEV protease cleavage site after the N-terminal $6 \times$ His tag. Meanwhile, full-length Lamtor 2 and Lamtor3 were subcloned into the two multiple cloning sites of the pACYCDuet-1 vector (Novagen), whereas full-length Lamtor4 and Lamtor5 (residues 83-173) were subcloned into the two multiple cloning sites of the pRSFDuet-1 vector (Novagen). None of these four proteins carry tags. The Ragulator protein complex was expressed in the Escherichia coli BL21(DE3) strain. Cells were grown at $37^{\circ}$ $\mathrm{C}$ in Luria Broth medium and induced when the optical density at $600 \mathrm{~nm}$ became 1.0 by adding $0.3 \mathrm{~mm}$ isopropyl thiogalactoside at $23{ }^{\circ} \mathrm{C}$ for $12 \mathrm{~h}$. The cultures were harvested by centrifugation and resuspended in $25 \mathrm{~mm}$ Tris- $\mathrm{HCl}, \mathrm{pH} 8.0,20 \mathrm{~mm}$ imidazole and $300 \mathrm{~mm} \mathrm{NaCl}$. The protein complex was first purified using the $\mathrm{Ni}^{2}$ ${ }^{+}$-NTA affinity chromatography, and the eluted protein was incubated with TEV protease $\left(1: 50\right.$ ratio, $\left.\mathrm{w} \mathrm{w}^{-1}\right)$ at $4{ }^{\circ} \mathrm{C}$ for $12 \mathrm{~h}$ to remove the $6 \times$ His tag in the presence of $\beta$-mercaptoethanol $\left(1: 1000\right.$ ratio, $\left.\mathrm{Vv}^{-1}\right)$. The protein was further purified by anion exchange chromatography, followed by gel filtration chromatography in $10 \mathrm{~mm}$ Tris- $\mathrm{HCl}, \mathrm{pH} 8.0,100 \mathrm{~mm} \mathrm{NaCl}$ and $2 \mathrm{~mm}$ dithiothreitol. Before crystallization, the Ragulator protein complex was concentrated by ultrafiltration to $15 \mathrm{mg} \mathrm{ml}^{-1}$. 
Crystallization was carried out at $287 \mathrm{~K}$ by using the hangingdrop vapor-diffusion method. Crystals were grown at $287 \mathrm{~K}$ after 1 week by mixing $1 \mu \mathrm{l}$ of protein with $1 \mu \mathrm{l}$ of crystallization solution containing $0.1 \mathrm{M}$ CHES (2-[ $N$-cyclohexylaminolethanesulphonic acid), $\mathrm{pH} 9.5,0.56 \mathrm{~m}$ sodium citrate tribasic and $1.4 \mathrm{~m}$ sodium chloride.

For purification of the Ragulator complex assembled with Lamtor1 C-terminal deletion or point mutation mutants, only Lamtor1 (residues 41-161) was replaced by either Lamtor1 (residues 41-150) or Lamtor1 (residues 41-161, $\mathrm{L}^{154} \mathrm{VVQF}^{158}$ mutated to $\mathrm{D}^{154} \mathrm{DDQD}{ }^{158}$ ), and other procedures remained the same.

\section{Data collection and structure determination}

Crystals were cryoprotected in the crystallization buffer supplemented with $25 \%$ glycerol. One set of diffraction data of the Lamtor4-Lamtor5 complex at $2.03 \AA$ resolution and one set of diffraction data of the Ragulator complex at $3.01 \AA$ resolution were collected at the beamline BL19U1 of National Center for Protein Sciences Shanghai at $100 \mathrm{~K}$ [25]. Data reduction was performed with the HKL3000 software (Charlottesville, VA, USA) [26]. Phases were determined using the Phaser program [27] in the CCP4 package [28]. Model building was performed using COOT [29], and refinement was performed by the REFMAC5 program [30] in the CCP4 package [28]. The final refined models have $R_{\text {work }} / R_{\text {free }}$ factors of $21.03 \% / 26.27 \%$ and $22.50 \% / 28.54 \%$ for the Lamtor4Lamtor5 and the Ragulator complexes, respectively. The quality of the structure models were checked with the CCP4 program PROCHECK [28], which showed good stereochemistry according to the Ramachandran plot.

\section{Purification of the RagA-RagC complex}

Human RagA (residues 6-302, with the point mutation T21N) and human $\mathrm{RagC}$ (residues 60-375, with the point mutation Q120L) were subcloned into the pETDuet-1 vector (Novagen) for recombinant protein expression in the Escherichia coli strain BL21 (DE3). RagA carries an N-terminal $6 \times$ His tag, whereas RagC has no tag. Bacteria cells were incubated at $37^{\circ} \mathrm{C}$ until the optical density (OD) at $600 \mathrm{~nm}$ reached 1.0. Protein expression was induced with $0.2 \mathrm{~mm}$ isopropyl thiogalactoside and the cells were further incubated at $16^{\circ} \mathrm{C}$ for $16-20 \mathrm{~h}$. The cultures were harvested by centrifugation and resuspended using $25 \mathrm{~mm}$ Tris- $\mathrm{HCl}, \mathrm{pH}$ 8.0, $20 \mathrm{~mm}$ imidazole and $300 \mathrm{~mm} \mathrm{NaCl}$. After lysis of bacterial cells with a cell homogenizer (JNBIO, Guangzhou, China) and centrifugation, the RagA-RagC protein complex was purified using $\mathrm{Ni}^{2+}$-NTA affinity chromatography and anion exchange chromatography, followed by gel filtration chromatography in $15 \mathrm{~mm}$ Tris- $\mathrm{HCl}, \mathrm{pH} 8.0,150 \mathrm{~mm} \mathrm{NaCl}$ and $4 \mathrm{~mm}$ dithiothreitol. The same procedure was used to purify the RagA-CTD (residues 183-302)-RagC-CTD (residues 239-375) protein complex.

\section{$\mathrm{Ni}^{2+}{ }_{-N T A}$ affinity chromatography assay between the}

Ragulator complex and the RagA-RagC GTPase complex

$\mathrm{Ni}^{2+}$-NTA affinity chromatography pull-down assay was performed using purified $6 \times$ His-tagged RagA (residues 6-302, T21N)-RagC (residues 60-375, Q120L) complex and untagged Ragulator complex, with the $6 \times$ His tag on Lamtor 1 already removed by the TEV protease. The $6 \times$ His-tagged RagA
(6-302, T21N)-RagC (60-375, Q120L) protein complex was diluted to $1 \mathrm{mg} \mathrm{ml}^{-1}$. The Ragulator complex was diluted to $0.5 \mathrm{mg} \mathrm{ml}^{-1}$. $\quad 6 \times$ His-tagged RagA $(6-302, \quad \mathrm{~T} 21 \mathrm{~N})-\mathrm{RagC}$ (60-375, Q120L) was first loaded onto the $\mathrm{Ni}^{2+}$-NTA affinity column. The flow-through was collected and the $\mathrm{Ni}^{2+}$-NTA affinity column was washed with 20 column volumes of equilibrium buffer (25 mM Tris- $\mathrm{HCl}, \mathrm{pH} 8.0,300 \mathrm{~mm} \mathrm{NaCl}$ and $20 \mathrm{~mm}$ imidazole). Untagged Ragulator was then loaded onto the $\mathrm{Ni}^{2+}$-NTA column and the flow-through was collected. The column was washed with another 20 column volumes of equilibrium buffer, and then eluted with 3 column volumes of the elution buffer (25 mm Tris- $\mathrm{HCl}, \mathrm{pH} 8.0,300 \mathrm{~mm} \mathrm{NaCl}$ and $300 \mathrm{~mm}$ imidazole). $\mathrm{Ni}^{2+}$-NTA affinity chromatography pull-down assay between $6 \times$ His-tagged RagA-CTD (residues 183-302)-RagC-CTD (residues 239-375) complex and untagged Ragulator complex was performed using the same method.

\section{Molecular graphics}

All the protein structure figures were generated by the PyMOL program [31].

\section{Accession codes}

The structural coordinates and structural factors have been deposited into the Protein data Bank (PDB) with codes of 5 YK3 and 5YK5 for human Ragulator complex and human Lamtor4Lamtor 5 complex, respectively.

\section{Conflict of Interest}

The authors declare no conflict of interest.

\section{Acknowledgements}

We thank Jianhua He, Wenming Qin, Lijie Wu and other staff at the beamline BL17U1 at Shanghai Synchrotron Radiation Facility (SSRF) and the beamline BL19U1 at National Center f or Protein Science Shanghai (NCPSS). This work was supported by grants from the National Natural Science Foundation of China (grant numbers 31470223 and 31670106), State Key Laboratory of Microbial Resources, Institute of Microbiology, Chinese Academy of Sciences and the Program for Professor of Special Appointment (Eastern Scholar) at Shanghai Institutions of Higher Learning.

\section{Author contributions}

Lamtor4-Lamtor5 and Ragulator protein complex purification, crystallization, and data collection were performed by ZM. RagA-RagC protein complex purification was performed by $\mathrm{LW}$. $\mathrm{Ni}^{2+}$ column pull-down assay was performed by ZM and LW. Size exclusion chromatography-multiangle light scattering assay was performed by ZM and WD. Crystal structure determination was performed by JW and GW. GW, ZM and LW wrote the paper. 


\section{References}

1 Wullschleger S, Loewith R, Hall MN. TOR signaling in growth and metabolism. Cell 2006; 124: 471-484.

2 Laplante M, Sabatini DM. mTOR signaling in growth control and disease. Cell 2012; 149: 274-293.

3 Saxton RA, Sabatini DM. mTOR signaling in growth, metabolism, and disease. Cell 2017; 168: 960-976.

4 Ben-Sahra I, Manning BD. mTORC1 signaling and the metabolic control of cell growth. Curr Opin Cell Biol 2017; 45: 72-82.

5 Bar-Peled L, Sabatini DM. Regulation of mTORC1 by amino acids. Trends Cell Biol 2014; 24: 400-406.

6 Dibble CC, Manning BD. Signal integration by mTORC1 coordinates nutrient input with biosynthetic output. Nat Cell Biol 2013; 15: 555-564.

7 Jewell JL, Russell RC, Guan KL. Amino acid signaling upstream of mTOR. Nat Rev Mol Cell Biol 2013; 14: 133-139.

8 Kim E, Goraksha-Hicks P, Li L, Neufeld TP, Guan KL. Regulation of TORC1 by Rag GTPases in nutrient response. Nat Cell Biol 2008; 10: 935-945.

9 Sancak Y, Peterson TR, Shaul YD et al. The Rag GTPases bind raptor and mediate amino acid signaling to $\mathrm{mTORC1}$. Science 2008; 320: 1496-1501.

10 Sancak Y, Bar-Peled L, Zoncu R, Markhard AL, Nada S, Sabatini DM. Ragulator-Rag complex targets mTORC1 to the lysosomal surface and is necessary for its activation by amino acids. Cell 2010; 141: 290-303.

11 Bar-Peled L, Schweitzer LD, Zoncu R, Sabatini DM. Ragulator is a GEF for the rag GTPases that signal amino acid levels to mTORC1. Cell 2012; 150: 1196-1208.

12 Dibble CC, Elis W, Menon S et al. TBC1D7 is a third subunit of the TSC1-TSC2 complex upstream of mTORC1. Mol Cell 2012; 47: 535-546.

13 Menon S, Dibble CC, Talbott G et al. Spatial control of the TSC complex integrates insulin and nutrient regulation of mTORC1 at the lysosome. Cell 2014; 156: 771-785.

14 Nada S, Hondo A, Kasai A et al. The novel lipid raft adaptor p18 controls endosome dynamics by anchoring the MEKERK pathway to late endosomes. EMBO J 2009; 28: 477-489.

15 Gong R, Li L, Liu Y et al. Crystal structure of the GtrlpGtr2p complex reveals new insights into the amino acidinduced TORC1 activation. Genes Dev 2011; 25: 1668-1673.

16 Loewith R, Jacinto E, Wullschleger S et al. Two TOR complexes, only one of which is rapamycin sensitive, have distinct roles in cell growth control. Mol Cell 2002; 10: 457-468.

17 Binda M, Péli-Gulli MP, Bonfils G et al. The Vam6 GEF controls TORC1 by activating the EGO complex. Mol Cell 2009; 35: 563-573.

18 Zhang T, Péli-Gulli MP, Yang H, De Virgilio C, Ding J. Ego3 functions as a homodimer to mediate the interaction between Gtr1-Gtr2 and Egol in the Ego complex to activate TORC1. Structure 2012; 20: 2151-2160.

19 Powis K, Zhang T, Panchaud N, Wang R, De Virgilio C, Ding J. Crystal structure of the Ego1-Ego2-Ego3 complex and its role in promoting Rag GTPase-dependent TORC1 signaling. Cell Res 2015; 25: 1043-1059.
20 Powis K, De Virgilio C. Conserved regulators of Rag GTPases orchestrate amino acid-dependent TORC1 signaling. Cell Discov 2016; 2: 15049.

21 Kurzbauer R, Teis D, de Araujo ME et al. Crystal structure of the p14/MP1 scaffolding complex: how a twin couple attaches mitogen-activated protein kinase signaling to late endosomes. Proc Natl Acad Sci USA 2004; 101: 10984-10989.

22 Lunin VV, Munger C, Wagner J, Ye Z, Cygler M, Sacher M. The structure of the MAPK scaffold, MP1, bound to its partner, p14. A complex with a critical role in endosomal map kinase signaling. $J$ Biol Chem 2004; 279: 23422-23430.

23 Jeong JH, Lee KH, Kim YM, Kim DH, Oh BH, Kim YG. Crystal structure of the Gtr1p(GTP)-Gtr2p(GDP) protein complex reveals large structural rearrangements triggered by GTP-to-GDP conversion. J Biol Chem 2012; 287 : 29648-29653.

24 de Araujo MEG, Naschberger A, Fürnrohr BG et al. Crystal structure of the human lysosomal mTORC1 scaffold complex and its impact on signaling. Science 2017; 358: 377-381.

25 Wang Q, Yu F, Huang S et al. The macromolecular crystallography beamline of SSRF. Nucl Sci Technol 2015; 26: 10102.

26 Otwinowski Z, Minor W. Processing of X-ray diffraction data collected in oscillation mode. Methods Enzymol 1997; 276: $307-326$.

27 McCoy AJ, Grosse-Kunstleve RW, Adams PD et al. Phaser crystallographic software. $J$ Appl Crystallogr 2007; 40: 658-674.

28 Collaborative Computational Project Number 4. The CCP4 suite: programs for protein crystallography. Acta Crystallogr D 1994; 50: 760-763.

29 Emsley P, Cowtan K. Coot: model-building tools for molecular graphics. Acta Crystallogr D 2004; 60: 2126-2132.

30 Winn MD, Murshudov GN, Papiz MZ. Macromolecular TLS refinement in REFMAC at moderate resolutions. Methods Enzymol 2003; 374: 300-321.

31 Schrodinger LLC. The PyMOL Molecular Graphics System, version 13r1. Schrodinger LLC, San Carlos, CA, USA, 2010.

(Supplementary Information is linked to the online version of the paper on the Cell Discovery website.)

This work is licensed under a Creative Commons Attribution 4.0 International License. The images or
other third party material in this article are included in the article's Creative Commons license, unless indicated otherwise in the credit line; if the material is not included under the Creative Commons license, users will need to obtain permission from the license holder to reproduce the material. To view a copy of this license, visit http://creativecommons.org/licenses/by/4.0/

(C) The Author(s) 2017 